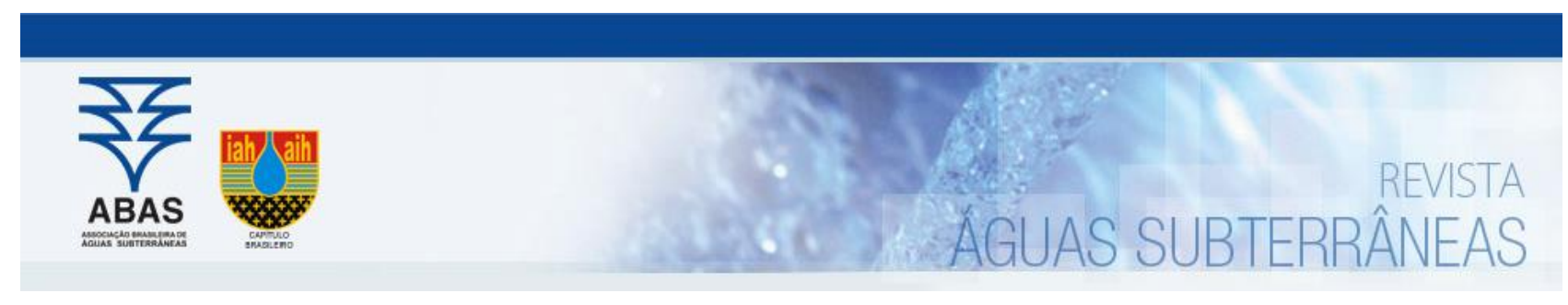

Artigos

\title{
Modelagem da favorabilidade à recarga das águas subterrâ- neas pelo paradigma da lógica difusa
}

\section{Groundwater recharge favorability modelling by diffuse logic paradigm}

\author{
Rodrigo Lilla Manzione1; César de Oliveira Ferreira Silva2; Claudiane Otília Paes ${ }^{2}$ \\ 1 Universidade Estadual Paulista “Julio de Mesquita Filho" - Faculdade de Ciências e Engenharia (UNESP/FCE), Tupã, SP \\ 2 Universidade Estadual Paulista "Julio de Mesquita Filho" - Faculdade de Ciências Agronômicas (UNESP/FCA), Botucatu, SP
}

$\triangle \underline{\text { rlmanzione@gmail.com, cesaroliveira.f.silva@gmail.com, claudianepaes@hotmail.com }}$

Palavras-chave:
Sistema Aquífero Guarani;
Análise espacial de dados geográficos;
Lógica fuzzy;
Mapeamento;
Inteligência artificial.

Keywords:

Guarani Aquifer System;

Geographical spatial data analysis;

Fuzzy logic;

Mapping;

Artificial intelligence.

Revisão por pares.

Recebido em: 14/04/2021.

Aprovado em: 29/07/2021.

\begin{abstract}
Resumo
As informações geográficas são imprecisas, o que significa que os limites entre fenômenos diferentes são confusos ou há heterogeneidade dentro de uma classe, devido a diferenças entre feições geológicas, pedológicas, geomorfológicas, vegetais e assim por diante. Métodos baseados em inteligência artificial (IA) fornecem soluções sensíveis à natureza imprecisa (fuzzy) do mundo real baseados em regras definidas por especialistas. A natureza incerta dos processos que controlam a recarga das águas subterrâneas em bacias hidrográficas permite que esses métodos possam ser aplicados na gestão das águas subterrâneas, auxiliando no planejamento e na tomada de decisão quanto ao uso da água e proteção de áreas vulneráveis. O objetivo deste trabalho foi definir áreas favoráveis para recarga das águas subterrâneas a partir de variáveis relacionadas amostradas próximas a poços de monitoramento em uma bacia hidrográfica em área de afloramento do Sistema Aquífero Guarani (SAG). Para isso, utilizou-se da lógica fuzzy para definir um sistema de inferência capaz de extrapolar espacialmente os dados amostrados pontualmente para toda a bacia hidrográfica, resultando em um mapa de favorabilidade à recarga baseado em variáveis relacionadas à textura e manejo do solo, feições de terreno e vegetação. 0 mapa síntese auxilia tanto o planejamento quanto a tomada de decisão do uso da terra considerando os processos hidrológicos em suas interfaces de superfície e subsuperfície. A partir dos resultados alcançados, estende-se a discussão sobre a importância das escolhas éticas nos processos de tomada de decisão em hidrogeologia relacionadas ao uso de métodos baseados em IA.
\end{abstract}

\section{Abstract}

Geographic information is uncertain, which means that the boundaries between different phenomena are blurred or there is heterogeneity within a class, due to differences between geological, pedological, geomorphological, vegetal features and so on. Methods based on artificial intelligence (Al) provide specific solutions to the fuzzy nature of the real world based on expert-knowledge. The uncertain nature of the processes that control groundwater recharge in watersheds allows these methods to be applied in groundwater management, supporting planning and decisionmaking related with water use and protection of vulnerable areas. The aim of this work was to define favourable areas for groundwater recharge from variables related variables samples near monitoring wells in a watershed in an outcrop area of the Guarani Aquifer System (GAS). Fuzzy logic was used to define an inference system capable of spatially extrapolating the point data for the entire watershed. The output was a map of favourability to recharge based on variables related to the texture and management of soil, terrain features and vegetation. The synthesis map support both planning and decision making on land use considering hydrological processes in its surface and subsurface interfaces. From the results achieved, the discussion on the importance of ethical choices in the hydrogeology decision-making processes related to the use of Al-based methods is extended.

DOI: http:/dx.doi.org/10.14295/ras.v35i2.30030

\section{INTRODUÇÃO}

A recarga das águas subterrâneas é uma variável crucial no planejamento e tomada de decisão em sistemas de recursos hídricos. Taxas, épocas, locais da recarga são importantes para uma gestão eficiente dos recursos hídricos subterrâneos, tanto em termos de abastecimento quanto contaminação da água. A recarga pode ser definida de forma geral como o fluxo de água atingindo o lençol freático, formando um acréscimo ao reservatório de água subterrânea a partir do mecanismo de percolação da água no solo (DE VRIES; SIMMERS, 2002). A partir da estimativa da recarga é possível planejar o uso e a ocupação das terras em função do seu potencial hídrico, ajustando o tipo de cultura à região, enfim, promover o uso sus- tentável da água, sem que a demanda afete a oferta (SCANLON et al., 2002; FEITOSA et al., 2008; MANZIONE, 2015). 0 entendimento da dinâmica das águas subterrâneas, tanto em termos de quantidade disponível para cada uma de suas funções como também aspectos quanto a sua qualidade dependem diretamente da estimativa relativas à recarga. Segundo Healy e Scanlon (2010), devido ao elevado número de variáveis e complexidade na modelagem de cada um desses processos do ciclo hidrológico envolvidos na recarga das águas subterrâneas, um modelo conceitual para a recarga das águas subterrâneas deve ser desenvolvido para seleção de métodos apropriados para sua estimativa. Modelos conceituais são simples conjuntos de hipóteses tipo como, onde, quando e porque a recarga ocorre na sua área de estudo. As hipóteses 
do método devem ser consistentes com o modelo conceitual, que pode ser refinado à medida que dados e análise vão se tornando disponíveis.

Os modelos de simulação hidrológica são usados para fornecer uma previsão ou projeção hidrológica, bem como uma meIhor compreensão dos processos do ciclo hidrológico (TUCCI, 2005; KRESIC; MIKSZEWSKI, 2013). Segundo KARAMOUZ et al. (2013), várias abordagens podem ser usadas para esse fim, sendo as saídas desses modelos uma representação conceitual simplificada de uma parte ou componente do ciclo hidrológico global. A complexidade dos sistemas estudados em geociências torna extremamente difícil impor as mesmas regras em locais diferentes, fazendo com que os sistemas especialistas baseados em inteligência artificial (IA) permaneçam nas sombras para muitas aplicações. Além disso, extensões de métodos de simulação para o contexto espacial ainda são pouco exploradas em relação às estimativas pontuais, já que envolvem dificuldades de amostragem e cobertura de heterogeneidades (DE GRUIJTER et al., 2006). Nestes procedimentos é aplicado um novo pensamento: deixar que os próprios dados geográficos assumam o protagonismo ao invés de deixar as estatísticas derivadas do conjunto de dados ditarem a análise, como matrizes de médias e covariâncias usadas nas classificações de máxima verossimilhança (JENSEN, 2015). Modelos inteligentes desenvolvidos para a tomada de decisões nas mais diversas áreas do conhecimento carregam consigo uma responsabilidade algorítmica ímpar, principalmente aqueles baseados em conceitos que trabalham com dados imprecisos, como a teoria dos conjuntos fuzzy. Os conjuntos fuzzy e sua lógica tornaram possível uma alternativa (em termos dos conjuntos clássicos padrão) e um tratamento formal para uma classe de dados cuja condição de verdade é definida em uma faixa numérica correspondente a diferentes graus de verdade. Esse recurso de um conjunto fuzzy torna a teoria dos conjuntos fuzzy apropriada para a representação de incerteza que não seja a forma probabilística. Esse conceito foi proposto pela primeira vez por Zadeh (1965) pela introdução da teoria dos conjuntos difusos, chamados conjuntos fuzzy daqui por diante. Na teoria dos conjuntos fuzzy, há uma transição gradual entre associação e não associação. Essa transição gradual da associação à teoria dos conjuntos fuzzy se deve à definição vaga dos limites dos conjuntos fuzzy.

No contexto da simulação determinística hidrológica, redes neurais artificiais (RNA), teoria dos conjuntos difusos (fuzzy), sistema de inferência fuzzy e sistema de inferência neuro-fuzzy adaptável são as principais aplicações (KARAMOUZ et al., 2013). Para os autores, os modelos baseados em lógica fuzzy são ferramentas poderosas para resolver problemas usando conceitos matemáticos muito simples, mas ressaltam que a lógica fuzzy não resolve novos problemas, e sim oferece novos métodos para resolver os problemas do dia a dia. Especifica- mente em águas subterrâneas, a teoria dos conjuntos fuzzy vem sendo aplicada a diferentes questões relacionadas com a qualidade e quantidade dos recursos hídricos. Mohamed et al. (2018) fizeram uma avaliação da qualidade da água subterrânea em uma bacia sedimentar não consolidada na China usando técnicas fuzzy. Duhalde et al. (2018) realizaram uma avaliação de base fuzzy da vulnerabilidade intrínseca da água subterrânea no Chile. Ghazavi et al. (2018) selecionou locais para poços de recarga artificiais em uma área urbana no Irã usando técnicas de lógica fuzzy. Nadiri et al. (2019) aplicou a lógica fuzzy para modelar as variações do nível do lençol freático na província do Azerbaijão Oriental, Irã. Já Das e Pal (2019) utilizaram logica fuzzy e análise AHP para determinar potenciais zonas de recarga na Índia.

O objetivo desse trabalho foi definir áreas favoráveis para recarga das águas subterrâneas utilizando um sistema de inferência fuzzy (SIF) definido a partir de variáveis relacionadas com os processos de infiltração e movimento da água no solo que regem a recarga, em uma bacia hidrográfica em área de afloramento do Sistema Aquífero Guarani (SAG).

\section{MATERIAIS E MÉTODOS}

\section{1. Área de estudo}

O presente estudo foi conduzido na Bacia do Ribeirão da Onça (BRO), no município de Brotas (SP), entre as latitudes $22^{\circ} 10^{\prime}$ e $22^{\circ} 15^{\prime}$ e longitudes $47^{\circ} 55^{\prime}$ e $48^{\circ} 00^{\prime}$ (Figura 1 ).

A BRO possui uma área aproximada de $65 \mathrm{~km}^{2}$ e a cobertura vegetal é composta por diversos cultivos como cana-de-açúcar, citros, eucalipto, pastagens, entre outras culturas. Segundo Wendland et al. (2007), trata-se de uma área de afloramento e recarga do Sistema Aquífero Guarani (SAG), e consequentemente, a bacia e o aquífero estão expostos a mecanismos potenciais de contaminação decorrente de atividades agrícolas. A BRO encontra-se assentada na sua maior parte sobre o arenito Botucatu, com solos formados a partir do intemperismo da Formação Botucatu e próximo ao exultório da bacia sobre o complexo Botucatu-diabásio, ambas unidades do Grupo São Bento de idade Mesozoica. Na maior parte da bacia a Formação Piramboia se encontra dezenas de metros abaixo da superfície do solo.

\subsection{Dados disponíveis}

A bacia possui uma rede de poços de monitoramento de nível freático que é realizado pela equipe do LHC/SHS/EESC/USP. Próximo aos poços, foi feito um levantamento de diversas características de solo resultando em um extenso banco de dados para análise. 
Figura 1 - Localização da área de estudo - Bacia do Ribeirão da Onça, Brotas (SP)

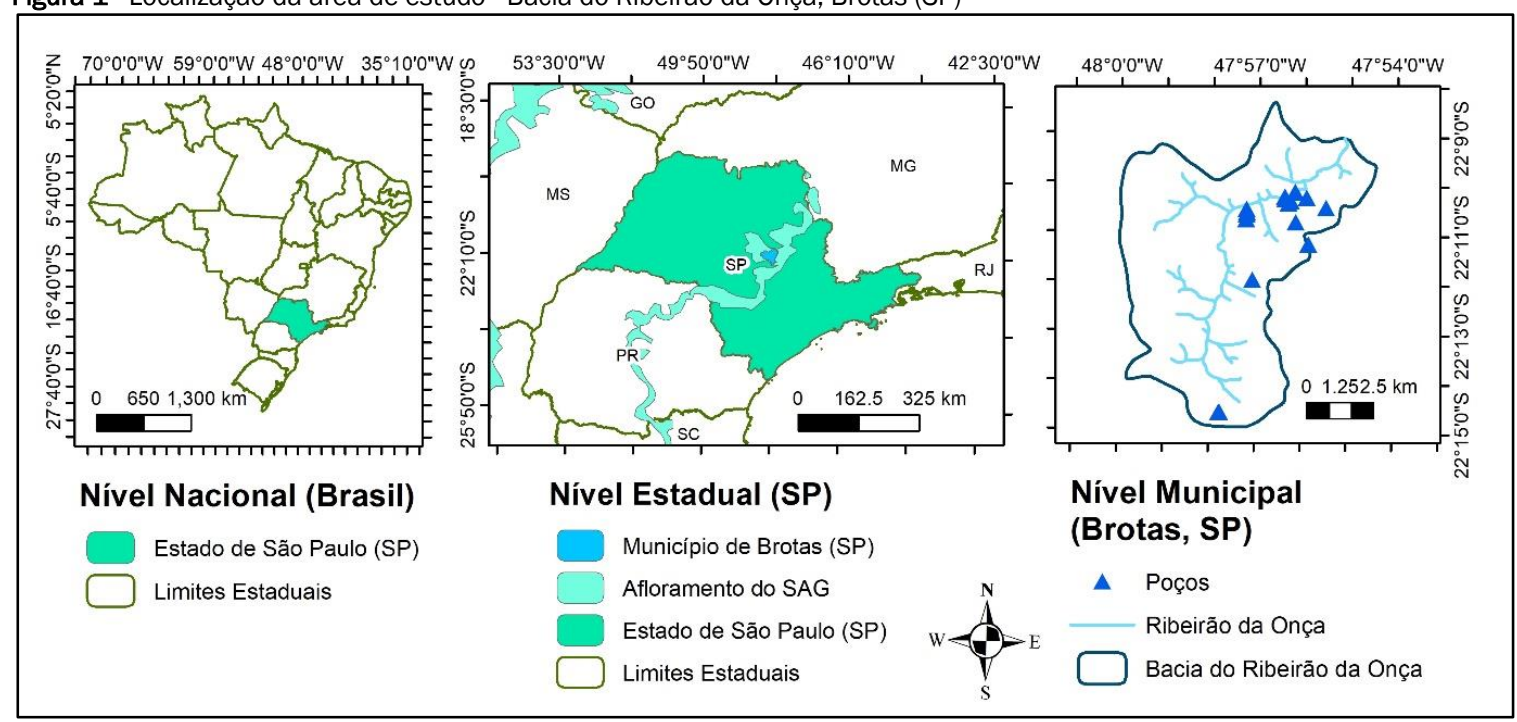

A seleção das variáveis utilizadas para criação do SIF baseouse em Manzione et al. (2015), que estudaram o mesmo conjunto de variáveis na BRO e verificaram que os principais fatores influenciando a variação dos níveis freáticos durante o período monitorado foi o tamanho da fração areia, atributos de terreno, textura do solo, manejo dos solos e das culturas, e vegetação. A análise para seleção das variáveis foi baseada em regressão linear múltipla. Para maiores detalhes quanto a seleção dessas variáveis recomendamos a leitura desse artigo.

Assim, para textura do solo selecionou-se a porcentagem de areia, para fração areia selecionou-se areia fina e areia muito fina, para atributos de terreno a distância à drenagem mais próxima, manejo dos solos a resistência a penetração, e para vegetação o coeficiente de cultura do uso atual do solo, totalizando 6 variáveis de entrada para o SIF.

Nesse estudo foram utilizadas propriedades físicas do solo como textura e granulometria, analisadas segundo os métodos da EMBRAPA (1997). A compactação do solo também foi medida através da resistência do solo à penetração, com o uso de um penetrômetro automático, da marca Falker, modelo PenetroLOG 1020, que executa medições até $60 \mathrm{~cm}$ de profundidade. Já dados sobre a distância à drenagem mais próxima foram obtidos a partir do modelo digital de terreno (MDT) da missão SRTM (Shuttle Radar Topography Mission) com resolução de 90 metros e o coeficiente de cultura (potencial evaporativo dado pela área foliar, arquitetura da planta, parte aérea, sistema radicular, cobertura vegetal e transpiração da cultura) tabulado segundo Allen et al. (1998) em razão do uso da terra no entorno de cada poço.

As relações entre essas propriedades e a recarga baseou-se em Healy e Scanlon (2010) e no conhecimento especialista dos autores sobre a área. Ressalta-se que essas relações podem ser alteradas e/ou invertidas conforme outras realidades locais e contextos hidrogeológicos se apresentem ao analista, não cabendo aqui um julgamento absoluto da validade dessas relações e sim como introduzi-las em um SIF para análise de recarga em bacias hidrográficas. Os valores redistribuídos entre 0 (desfavorável à recarga) e 1 (favorável à recarga) utilizaram como limites das funções de pertinência fuzzy os seguintes valores apresentados na Tabela 1.

Tabela 1 - Limites fuzzy estabelecidos a partir de conhecimento especialista

\begin{tabular}{lcc}
\hline \multicolumn{1}{c}{ Variável } & 0 & 1 \\
\hline Areia (\%) & $<85$ & $>85$ \\
Areia fina (\%) & $<40$ & $>40$ \\
Areia muito fina (\%) & $<20$ & $>20$ \\
Resistência à penetração $(\mathrm{kPa})$ & $>2.000$ & $<2.000$ \\
Distância à drenagem $(\mathrm{m})$ & $<500$ & $>500$ \\
Coeficiente de cultura $(-)$ & $>1$ & $<1$ \\
\hline
\end{tabular}

\subsection{Sistema de inferência fuzzy e mapeamento da favorabili- dade a recarga}

A lógica utilizada neste trabalho é um sistema de representação e inferência de informações imprecisas para fins de tomada de decisões por meio da teoria dos conjuntos fuzzy. 0 SIF foi construído com base no conhecimento de especialistas, ou seja, dependente do conhecimento de quem entende o sistema, no caso, o hidrogeólogo. No entanto, o SIF é um sistema muito flexível. Um SIF pode ser facilmente modificado adicionando ou excluindo regras sem a necessidade de criar um novo SIF. Além disso, dados imprecisos podem ser usados no SIF (mas não funcionam com incerteza) devido ao uso de elementos em um conjunto fuzzy (valores de pertinência). Uma vantagem importante do SIF é que ele pode ser usado junto com outros métodos clássicos. O SIF seguiu o método de Mamdani (MAMDANI; ASSILIAN, 1975). O método de Mamdani é o SIF mais comum usado para fins práticos, uma vez 
que é apropriada para um pequeno número de variáveis, e usa a estrutura simples de operações "min-max". Este sistema inclui as seguintes etapas:

- $\quad$ Etapa 1 - Avaliação de cada regra antecedente: 0 primeiro passo é a "distorção de entrada", onde são obtidos os valores de pertinência correspondentes dos valores de entrada fornecidos. Se o antecedente da regra tiver mais de uma parte, um operador difuso (IF - THEN) será aplicado para obter um único valor de pertinência. Se ambas as partes do antecedente foram unidas por uma conjunção ("e"), uma operação AND foi empregada e o valor mínimo seria selecionado. Nesse estudo, determinou-se valores limites de cada variável em estudo que seriam desfavoráveis (valor 0 ) ou favoráveis (valor 1) para a recarga das águas subterrâneas baseado no operador IF - THEN.

- $\quad$ Etapa 2 - Obtenção a conclusão de cada regra: Com base no consequente dado de cada regra (um conjunto fuzzy) e no valor antecedente obtido na Etapa 1, um ope- rador de implicação fuzzy é aplicado para obter um novo conjunto fuzzy. Dois métodos de implicação foram utilizados: o mínimo, que trunca a função de pertinência do consequente, e o produto, que a escala.

- $\quad$ Etapa 3 - Conclusões agregadas: Nesta etapa, o operador de agregação fuzzy (soma) é usado para combinar as saídas obtidas (funções de pertinência) para cada regra da Etapa 2 em um único conjunto fuzzy.

Para estudo, não foi realizada a etapa de Desfuzzyficação para que os valores entre 0 e 1 fossem mantidos para cada mapa. A partir daí os valores fuzzy variando de 0 a 1 para cada variável foram reunidos em um mapeamento de síntese em sistema das informações geográficas onde por meio de operadores booleanos e álgebra de mapas gerou-se um índice de favorabilidade de recarga. Todos os cálculos foram realizados com a ferramenta Matlab do software Mathworks com o Fuzzy Logic Toolbox e o sistema de informações geográficas QGIS. A Figura 2 resume a metodologia proposta.

Figura 2 - Proposta metodológica para desenvolvimento de um Sistema de inferência Fuzzy para mapeamento de áreas favoráveis à recarga das águas subterrâneas

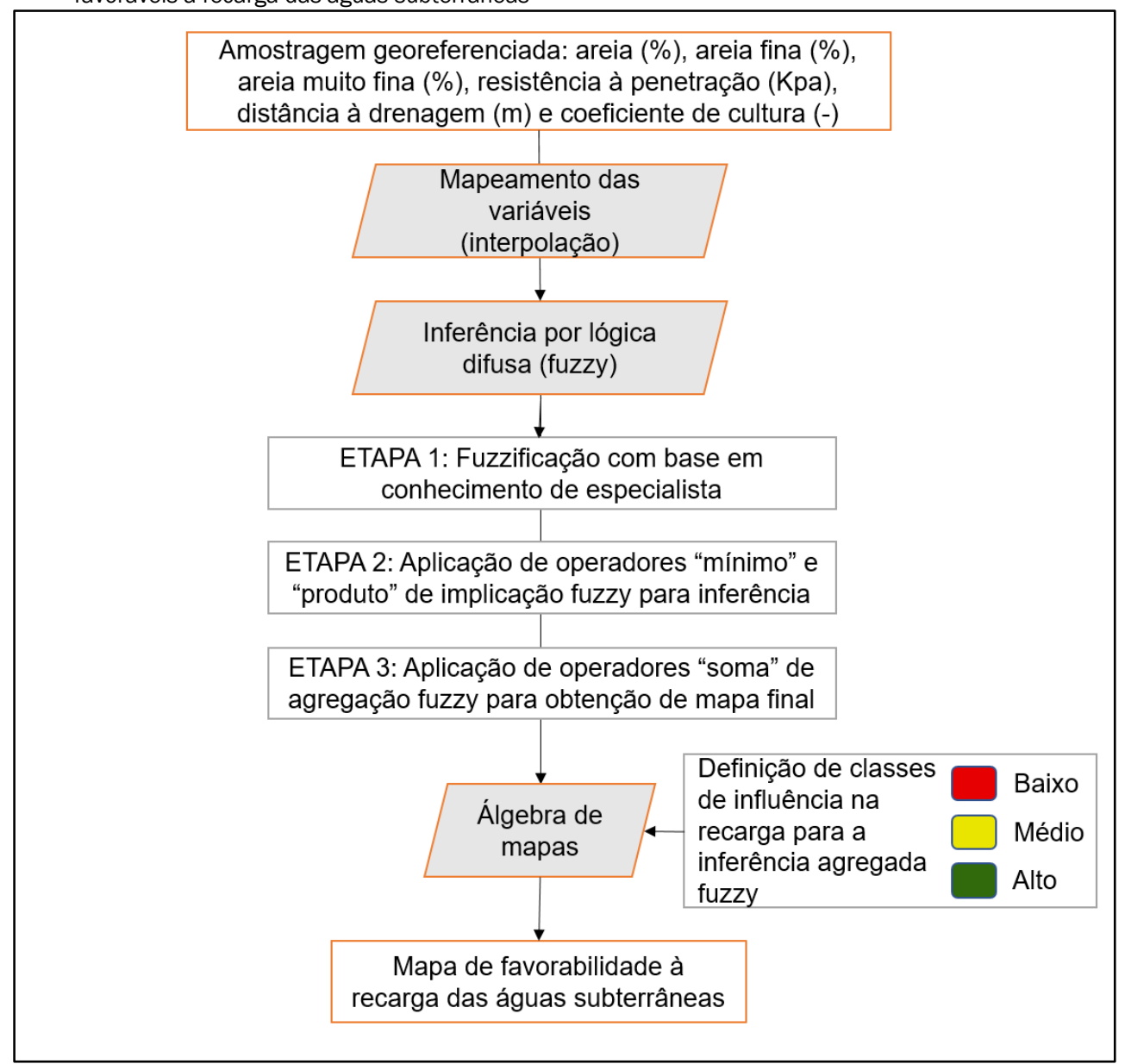

\section{RESULTADOS E DISCUSSÕES}

As variáveis envolvidas nos SIF estabelecido foram mapeadas conforme apresentado na Figura 3. Posteriormente, as variá- veis foram fuzzyficadas, resultando em mapas escalonados de 0 a 1, de acordo com a desfavorabilidade/favorabilidade da área à recarga em relação àquela variável (Figura 4). 
Areia (\%)

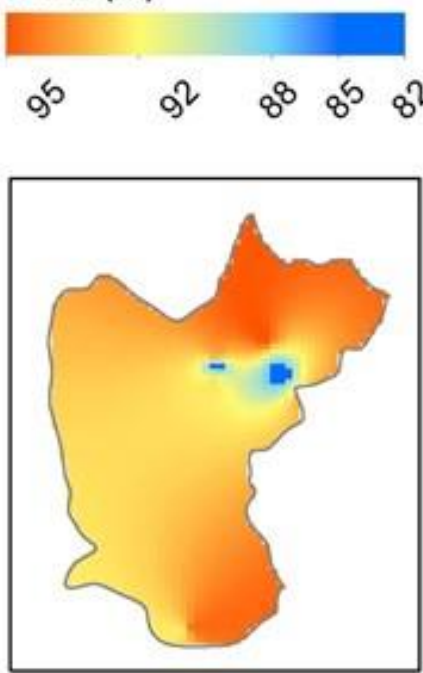

RP (kPA)
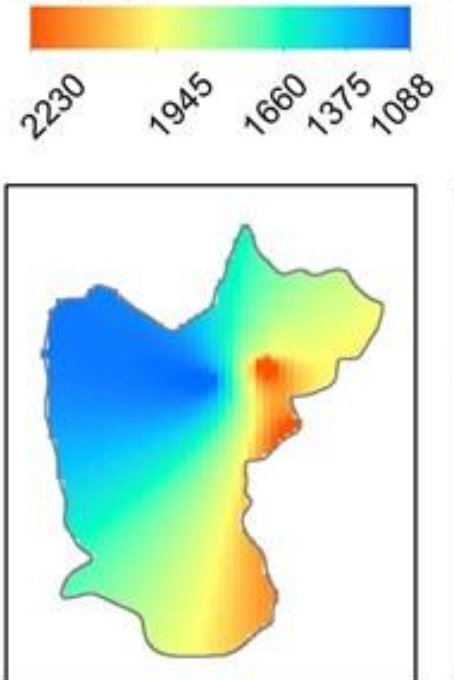

0

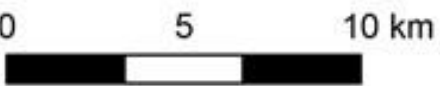

Areia Fina $(\%)$
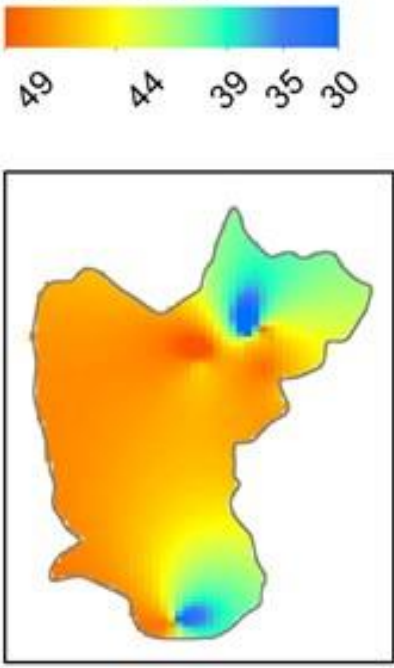

Distância de Drenagem ( $m$
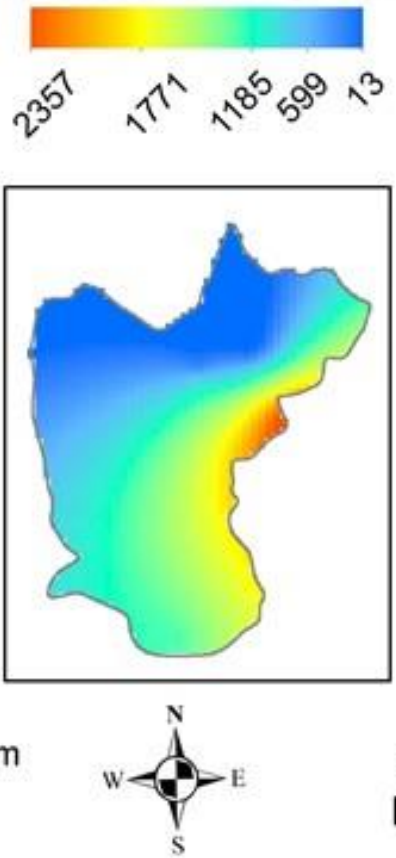

Areia Muito Fina (\%)
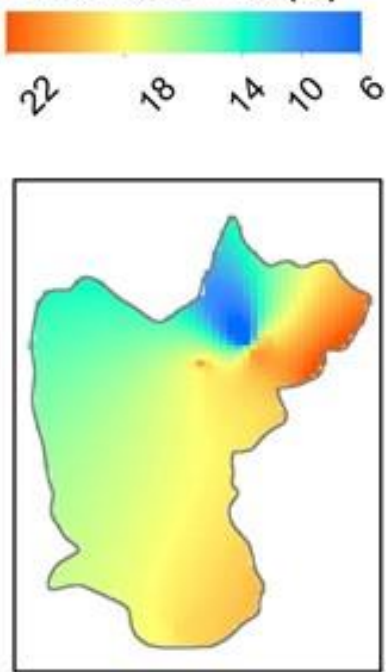

Coeficiente de Cultivo
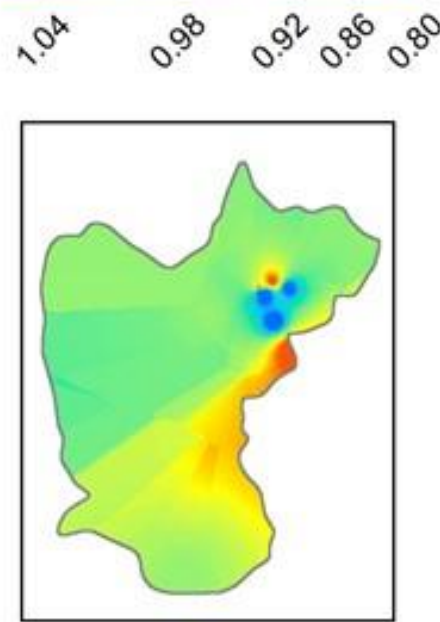

\section{Projeção Geodésica} Datum SIRGAS 2000
Dessa forma, a variável areia foi classificada de 0 a 1, ou seja, quanto maior a porcentagem de areia na bacia maior será a recarga, da mesma forma para as frações de areia fina e muito fina forem maiores. No mapa fuzzyficado de areia verifica-se maior influência desta variável a nordeste e ao sul da bacia, locais com maior ocorrência de areia. Apesar de que uma quantidade de areia prejudica a retenção de água no solo, considerou-se que altas porcentagens de areia teriam uma influência positiva para a recarga já que a permeabilidade do solo seria maior (REY-NOLDS; ELRICK, 1986). Os mapas fuzzyficados de areia fina e areia muito fina seguiram a mesma Iógica: a areia fina ocorre majoritariamente na parte central da região nordeste e ao sul da bacia, enquanto areia muito fina concentra-se na parte sudeste da bacia. 0 mapa da distância de drenagem acompanha as variações de terreno, sendo as áreas mais distantes das drenagens principais, próximas aos divisores de água mais favoráveis a recarga enquanto que locais nos fundos de vale onde a zona vadosa é menor seriam menos propícios à recarga. Essa relação entre o relevo e o nível da água subterrânea na BRO foi descrita por Manzione et al. (2012). A margem direita do Ribeirão da Onça apresenta uma maior densidade de drenagem, fazendo com que seja considerada uma área pior para recarga em relação a margem esquerda. Para esse mapa adotou-se a escala de zero a um com um limite de 500 metros. Os mapas de resistência do solo a penetração e coeficiente de cultura foram fuzzyficados de maneira inversa, já que os maiores valores influenciam negativamente a recarga: o solo apresenta altos valores de resistência a penetração na porção noroeste da bacia, sendo áreas com alto grau de compactação que dificultam 
a infiltração de água no solo e favorecem a erosão devido ao aumento do escoamento superficial. 0 coeficiente de cultura mostra uma menor influência na região central e ao norte da bacia, onde não estão presentes cultivos de eucalipto e cana de açúcar em detrimento de pastagens e outros cultivos pere- nes. Nas áreas onde os cultivos apresentam maior consumo hídrico a interceptação radicular interferiria negativamente na recarga, não permitindo que a água infiltrada alcançasse a zona saturada do solo.

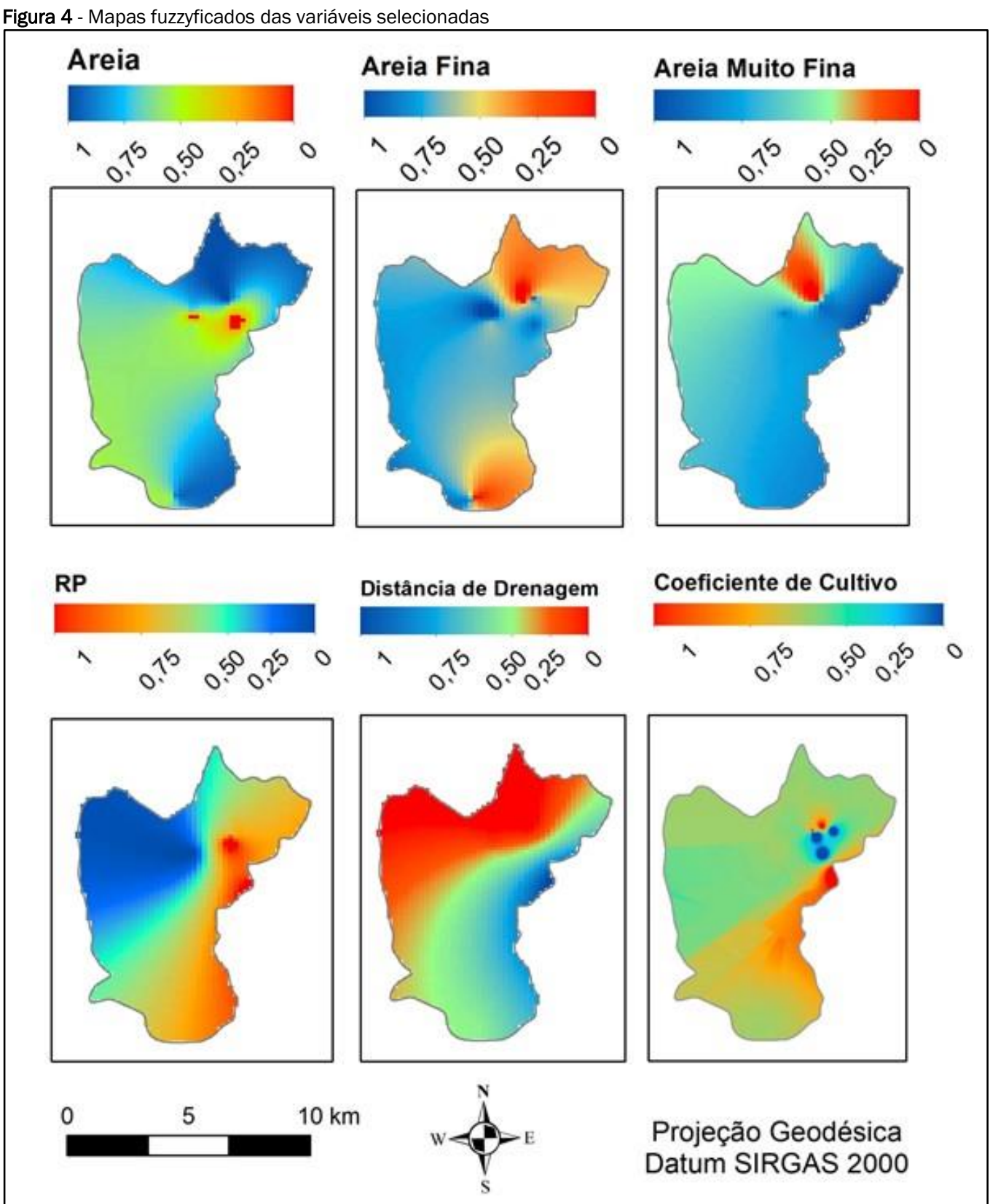

Com as variáveis todas fuzzyficadas, foi realizado um mapeamento de síntese através da álgebra de mapas e, posteriormente, reclassificando as áreas mais ou menos favoráveis à recarga na Bacia do Ribeirão da Onça conforme apresentado na Figura 5. A soma dos scores de 0 a 1 de todos os mapas resultaria em áreas mais favoráveis a recarga com maiores notas e menos favoráveis com menores notas. As notas variaram de 0,2 a 2,2 , sendo o potencial de recarga considerado baixo entre 0 e 1 , médio entre 1 e 2 e alto superior a 2. 
Figura 5 - Áreas prioritárias de recarga na Bacia do Ribeirão da Onça, Brotas (SP) calculadas pela lógica fuzzy

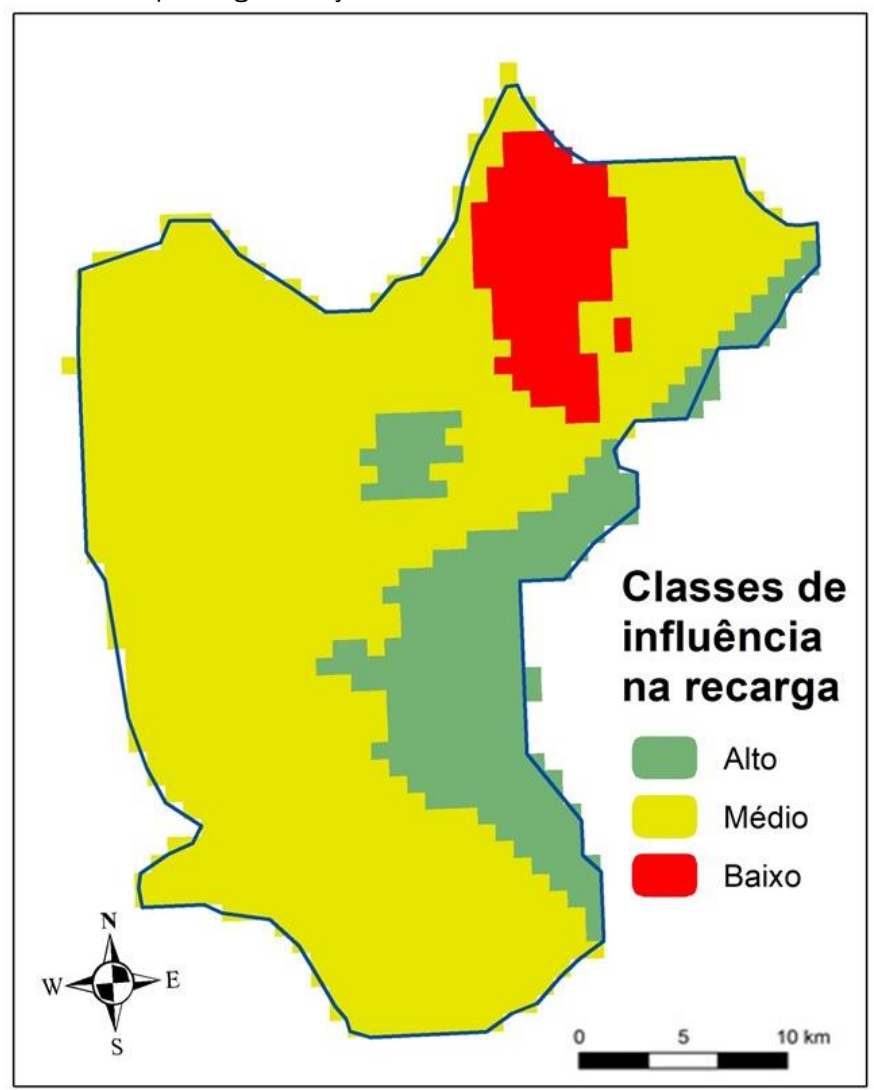

A Tabela 2 indica a porcentagem da área ocupada por cada classe definida na bacia e sua respectiva área em hectares. Podemos observar no mapa de potencial de recarga que as áreas mais favoráveis foram classificadas no centro e à leste da bacia (próximo ao divisor de águas). Nas porções noroeste, oeste e sul da bacia observa-se uma área com médio potencial de recarga, enquanto que a área ao norte foi classificada com baixo potencial de recarga. A classe baixo potencial indica lugares onde há uma influência negativa dessas variáveis no processo de recarga, a classe médio potencial indica áreas onde essas características não prejudicam a recarga de forma acentuada (porém requerem uma maior atenção quanto ao manejo do uso da terra e das culturas, para que não ocorram maiores influências) e a classe alto potencial indica áreas onde as características naturais, juntamente com aspectos de uso do solo, e interação rio-aquífero apresentam influência positiva para a recarga.

Tabela 2 - Área percentual e relativa de cada classe de favorabilidade a recarga

\begin{tabular}{lccc} 
Tabela 2 - Área percentual e relativa de cada classe de favorabilidade a recarga & \\
\hline & Classe & $\%$ & ha \\
\hline Alto & & 17,40 & 1.107 \\
Médio & 74,80 & 4.767 \\
Baixo & 7,80 & 491 \\
\hline Total & 100 & 6.365 \\
\hline
\end{tabular}

Utilizando como base o uso da terra na ocasião das amostragens (TANIKAWA; MANZIONE, 2011), observa-se que as áreas em que a favorabilidade à recarga é baixa são aquelas onde a ocupação da terra se dá por cultivos de cana-de-açúcar, enquanto nas áreas onde o uso da terra se dá por reflorestamentos por eucalipto o potencial de recarga é médio e alto e por citros irrigados no centro da bacia também é alto. Dedecek e Gava (2005) apontam que apesar de áreas onde houve plantio recente terem exigido tráfego intenso de máquinas para operação, a camada superficial do solo é mobilizada para plantio, tornando a compactação na camada superficial me- nor. Entretanto, com o desenvolvimento da cultura, outros processos que ocorrem no solo até a chegada da planta à fase adulta podem influenciar de forma negativa a recarga, sendo importante considerar as fases de desenvolvimento da cultura ao avaliar a sua influência na recarga. Laudos e estudos ambientais, quando tratam da utilização de recursos naturais, como solo e água, devem compreender o cenário na sua totalidade, entendendo que há um equilíbrio dinâmico no meio, e que toda ação realizada em superfície, tem uma resposta direta nas águas subterrâneas (PAES, 2012). 
O mapa de favorabilidade à recarga pode ser utilizado no planejamento do uso da terra em áreas frágeis como as de afloramento do SAG, indicando locais possuam maior favorabilidade natural à recarga. Assim, pode-se definir a capacidade de uso da terra, não somente em relação aos recursos naturais superficiais, mas também as águas subterrâneas, indicando os tipos de cultivos em relação a sua exigência de manejo, consumo hídrico e influências no meio físico. Em locais em que o uso da terra não foi executado com prévio planejamento, ferramentas como o modelo utilizado nesse estudo e os mapas gerados a partir dele podem subsidiar estratégias de gestão, apontando as áreas que necessitam de maior atenção no manejo para evitar influências negativas na área de recarga. O mapa de favorabilidade à recarga também pode ser utilizado para identificar regiões que necessitem de recuperação em áreas de afloramento de aquífero que foram degradadas, evitando generalizações e empregando corretamente as técnicas de remediação.

Uma vez estabelecidas e delineadas as relações entre as variáveis em estudo e a recarga no sentido prático, resta a questão de como esses resultados devem ser interpretados no sentido filosófico, uma vez que se tratam de estimativas realizadas utilizando um método baseado em IA e interferência especialista. Os geocientistas devem estar cientes de suas responsabilidades ao usar métodos de IA e as implicações de suas escolhas. Mas como Morone e Peppoloni (2017) apontaram sobre dilemas éticos em geociências: podemos perguntar, mas podemos responder? Como Manzione e Matulovic (2021) salientam, a responsabilidade ética na utilização de métodos de IA deve ser global e distribuída, pois cada agente ou organização escolhe um caminho em detrimento dos demais, de acordo com os valores intrínsecos a cada um deles. Precisa ser global pela amplitude que as questões morais podem ter em função da inserção das tecnologias informacionais na conduta dos agentes humanos, e distribuídas entre todos os atores da cadeia (SILVA et al., 2021).

Portanto, observa-se que o modelo desenvolvido tem grande potencial no estudo do fenômeno da recarga. Quando se busca, modelar variáveis relacionadas ao solo, sabe-se que há grande variabilidade nos valores coletados, uma vez que o solo muda muito em pequenas distâncias, e isso influencia os parâmetros estatísticos encontrados. Portanto, ao analisar um modelo que investigue a influência de variáveis do solo na recarga subterrânea, a expertise do profissional é de grande importância, para avaliar não somente os parâmetros estatísticos do modelo, mas também seu sentido físico para determinar e responder um fenômeno.

\section{CONCLUSÕES}

A partir do modelo proposto e dos resultados obtidos, pode-se verificar que a Bacia do Ribeirão da Onça possui favorabilidade média para recarga em $74,80 \%$ de sua área com base nos parâmetros texturais e características de uso e manejo do solo, além da distância da drenagem mais próxima. 0 componente espacial do modelo fuzzy permitiu estimar áreas prioritárias para recarga em toda bacia hidrográfica apesar de um conjunto limitado de dados. Os resultados permitem a delineação de áreas prioritárias de recarga visando a conservação dos recursos naturais, planejamento e ordenamento territorial, elaboração de cartas de vulnerabilidade e projetos de gerenciamento artificial da recarga. Destaca-se que o SIF pode ser realimentado com novos dados para atualização e readequação contínua do modelo. Recomenda-se ainda atenção ao extrapolar os resultados encontrados para outras áreas de estudo, uma vez que as regras apresentadas pelo SIF são relativas aos dados e ao contexto hidrogeológico local.

\section{AGRADECIMENTOS}

Os autores agradecem a FAPESP - Fundação de Amparo à Pesquisa do Estado de São Paulo pelas bolsas e auxílios concedidos para execução dessa pesquisa (Processos \# 2009/05204-8, 2011/07412-7, 2011/11484-3, 2012/07703-4).

À equipe do LCH/SHS/EESC/USP (Convênio 079/2010 UNESP/USP Processo 285/100/01/2008) pelo apoio logístico e compartilhamento dos dados.

\section{REFERÊNCIAS}

ALLEN, R. G.; PEREIRA, L. S.; RAES, D.; MARTIN, S. Crop evapotranspiration. FAO, Irrigation and Drainage Paper, v. 56. Rome: FAO, 1998. 297 p.

DAS, B.; PAL, S. C. Combination of GIS and fuzzy-AHP for delineating groundwater recharge potential zones in the critical Goghat-II block of West Bengal, India. HydroResearch, v. 2, p. 21-30,

https://doi.org/10.1016/j.hydres.2019.10.001

2019.

DE VRIES, J. J.; SIMMERS, I. Groundwater recharge: an overview of processes and challenges. Hydrogeology Journal, v. 10 , n. 1, p. 5-17, 2002. https://doi.org/10.1007/s10040$\underline{001-0171-7}$

DEDECEK, R. A; GAVA, J. L. Influência da compactação do solo na produtividade da rebrota de eucalipto. Revista Árvore, v. 29, n. 3, p. 383-390, 2005. https://doi.org/10.1590/S0100$\underline{67622005000300005}$

DE GRUIJTER, J. J.; BRUS, D. J.; BIERKENS, M. F. P.; KNOTTERS, M. Sampling for Natural Resource Monitoring. Berlin: Springer, 2006. 334 p. https://doi.org/10.1007/3-540-33161-1

DUHALDE, D. J.; ARUMÍ, J. L.; OYARZÚN, R. A.; RIVERA, D. A. Fuzzy-based assessment of groundwater intrinsic vulnerability of a volcanic aquifer in the Chilean Andean Valley. Environmental Monitoring Assessment, v. 190, 390, 2018. https://doi.org/10.1007/s10661-018-6758-4

EMBRAPA (Empresa Brasileira de Pesquisa Agropecuária). Manual de métodos de análise de solos. 2. ed. Rio de Janeiro: EMBRAPA Solos, 1997. 212 p.

FEITOSA, E. C.; MANOEL FILHO, J.; COSTA, W. D.; FEITOSA, F. A. C.; DEMETRIO, J. G. A.; FRANÇA, H. P. M. Avaliação de recursos hídricos subterrâneos. In: FEITOSA, F. A. C.; MANOEL FILHO, J.; FEITOSA, E. C.; DEMETRIO, J. G. A. Hidrogeologia: conceitos e aplicações. Rio de Janeiro: CPRM/LABHID, 2008. p. 661-671. 
GHAZAVI, R.; BABAEI, S.; ERFANIAN, M. Recharge wells site selection for artificial groundwater recharge in an urban area using fuzzy logic technique. Water Resources Management, v. 32, 3821, 2018. https://doi.org/10.1007/s11269-0182020-7

HEALY, R.; SCANLON, B. Estimating groundwater recharge. Cambridge: Cambridge University Press, 2010. 256 p. https://doi.org/10.1017/CB09780511780745

JENSEN, R. Introductory digital image processing: a remote sensing perspective. 4. ed. Upper Saddle River: Prentice Hall Press, 2015. $544 p$

KARAMOUZ, M. ; NAZIF, S. ; FALAHI, M. Hydrology and hydroclimatology. Principles and applications. Boca Raton: CRC Press, 2013. 742 p. https://doi.org/10.1201/b13771

KRESIC, N.; MIKSZEWSKI, A. (Eds.). Hydrogeological conceptual site models: data analysis and visualization. Boca Raton: CRC Press, 2013. 600 p. https://doi.org/10.1201/b12151

MAMDANI, E. H.; ASSILIAN, S. An experiment in linguistic synthesis with a Fuzzy Logic Controller. International Journal of Man-Machine Studies, v. 7, n. 1, p. 1-13, 1975. https://doi.org/10.1016/S0020-7373(75)80002-2

MANZIONE, R. L. Águas subterrâneas: conceitos e aplicações sob uma visão multidisciplinar. Jundiaí: Paco Editorial, 2015. $388 \mathrm{p}$.

MANZIONE, R. L.; WENDLAND, E.; TANIKAWA, D. H. Stochastic simulation of time-series models combined with geostatistics to predict water-table scenarios in a Guarani Aquifer System outcrop area, Brazil. Hydrogeology Journal, v. 20, n. 7, p. 1239-1249, 2012. https://doi.org/10.1007/s10040-0120885-8

MANZIONE, R. L.; SOLDERA, B. C.; PAES, C. O.; WENDLAND, E. Analyses of water table depths variations in an outcrop area of the Guarani aquifer system in Brotas/SP-Brazil. IRRIGA, Edição Especial, 20 anos Irriga + 50 anos FCA, p. 186-197, 2015. https://doi.org/10.15809/irriga.2015v1n1p186

MANZIONE, R. L.; MATULOVIC, M. Decision-Making in Groundwater Management: Where Artificial Intelligence Can Really Lead Geoscientists? In: ABRUNHOSA, M.; CHAMBEL, A.; PEPPOLONI, S.; CHAMINÉ, H. I. (Eds.). Advances in Geoethics and Groundwater Management: Theory and Practice for a Sustainable Development. Berlin: Springer, p. 441-445, 2021. https://doi.org/10.1007/978-3-030-59320-9 93

MOHAMED, A. K.; LIU, D.; MOHAMED, M. A. A.; SONG, K. Groundwater quality assessment of the quaternary unconsolidated sedimentary basin near the Pi river using fuzzy evaluation technique. Applied Water Science, v. 8, n. 65, 2018. https://doi.org/10.1007/s13201-018-0711-0
MARONE, E.; PEPPOLONI, S. Ethical dilemmas in geosciences. We can ask, but, can we answer? Annals of Geophys-Italy, v. 60, n. 7, AG-7445, 2017. https://doi.org/10.4401/ag-7445

NADIRI, A. A.; NADERI, K.; KHATIBI, R.; GHAREKHANI, M. Modelling groundwater level variations by learning from multiple models using fuzzy logic. Hydrological Science Journal, v. 64 $\begin{array}{llll}\text { n. } 2, & \text { p. } & 210-226, & \end{array}$ https://doi.org/10.1080/02626667.2018.1554940

PAES, C. O. Influência do uso e manejo dos solos nos mecanismos de recarga das águas subterrâneas em uma bacia hidrográfica em área de afloramento do Sistema Aquífero Guarani (SAG). Trabalho de Conclusão de Curso (Graduação em Geografia) - UNESP/Campus Experimental de Ourinhos, 110 p. 2012

REYNOLDS, W. D.; ELRICK, D. E. A method for simultaneous in situ measur ements in the vadose zone of field satured hydraulic conductivity, sorptivity and the conductivity - pressure head relationship. Ground water monitoring review, v. 6, n. 1, p. 84 95, 1986. https://doi.org/10.1111/i.1745 6592.1986.tb01229.x

SILVA, C. O. F.; MATULOVIC, M.; MANZIONE, R. L. New dilemmas, old problems: advances in data analysis and its geoethical implications in groundwater management. SN Applied Sciences, v. 3, n. 607, 2021. https://doi.org/10.1007/s42452021-04600-w

SCANLON, B. R., HEALY, R. W.; COOK, P. G. Choosing appropriate techniques for quantifying groundwater recharge. Hydrogeology Journal, v. 10, n. 1, p. 18-39, 2002. https://doi.org/10.1007/s10040-001-0176-2

TANIKAWA, D. H.; MANZIONE, R. L. Análise da correlação entre a resposta espectral da cultura de eucalipto e oscilação de níveis freáticos em área de recarga do Sistema Aquífero Guarani. In: CONGRESSO DE INICIAÇÃO CIENTÍFICA DA UNESP, XXIII, 2011, São Pedro. [Anais...] São Pedro: UNESP, 2011.

TUCCI, C. E. M. Modelos hidrológicos. 2. ed. Porto Alegre: Associação Brasileira de Recursos Hídricos / Editora da UFRGS, 2005. 678 p.

WENDLAND, E.; BARRETO, C. E. A. G.; GOMES, L. H. Water balance in the Guarani Aquifer outcrop zone based on hydrogeologic monitoring. Journal of Hydrology, v. 342, p. 261-269, 2007. https://doi.org/10.1016/i.jhydrol.2007.05.033

ZADEH, L. A. Fuzzy sets. Information and control, v. 8, n. 3, p. 338-353, $\quad 1965 . \quad$ https://doi.org/10.1016/S00199958(65)90241-X 